\title{
Outpatient High-Dose-Rate Mold Brachytherapy for Lower Gingival Squamous Cell Carcinoma: A First Report
}

\author{
Ami Takeshita ${ }^{1}$, Hiroaki Shimamoto ${ }^{1,2, *}$, Yuka Uchimoto ${ }^{1}$, Tomomi Tsujimoto ${ }^{1}$, Tetsuo Miyamoto ${ }^{3}$, \\ Sven Kreiborg 4,5, Sanjay M. Mallya 6, Michio Oda 7, Takero Hirata ${ }^{2}$, Kazuhiko Ogawa ${ }^{2}$, Hiroya Shiomi ${ }^{1,2}$ \\ Shumei Murakami 1,2
}

1 Department of Oral and Maxillofacial Radiology, Osaka University Graduate School of Dentistry, 1-8 Yamadaoka, Suita, Osaka 565-0871, Japan; takeshita-a@dent.osaka-u.ac.jp (A.T.); uchimoto@dent.osakau.ac.jp (Y.U.); tomo-mac@dent.osaka-u.ac.jp (T.T.); h.shiomi@gmail.com (H.S.) shumei@dent.osaka-u.ac.jp (S.M.)

2 Department of Radiation Oncology, Osaka University Graduate School of Medicine, 2-2 Yamadaoka, Suita, Osaka 565-0871, Japan; hirata@radonc.med.osaka-u.ac.jp (T.H.); kogawa@radonc.med.osaka-u.ac.jp (K.O.)

3 Dental Laboratory, Osaka University Dental Hospital, 1-8 Yamadaoka, Suita, Osaka 565-0871, Japan; miyamoto@dent.osaka-u.ac.jp

4 Department of Pediatric Dentistry and Clinical Genetics, School of Dentistry, Faculty of Health and Medical Sciences, University of Copenhagen, Nørre Allé 20, 2200, Copenhagen, Denmark; skrei@sund.ku.dk

5 3D Craniofacial Image Research Laboratory (School of Dentistry, University of Copenhagen; Centre of Head and Orthopedics, Copenhagen University Hospital Rigshospitalet; and Department of Applied Mathematics and Computer Science, Technical University of Denmark), Nørre Allé 20, 2200, Copenhagen, Denmark

6 Section of Oral and Maxillofacial Radiology, UCLA School of Dentistry, 10833 Le Conte Ave., Los Angeles, CA 90095-1668, USA; smallya@dentistry.ucla.edu

7 Department of Medical Technology, Osaka University Hospital, 2-15 Yamadaoka, Suita, Osaka 565-0871, Japan; oda@radonc.med.osaka-u.ac.jp

* Correspondence: h-shima@dent.osaka-u.ac.jp; Tel.: +81-6-6879-2967; Fax: +81-6-6879-2970

\begin{abstract}
Brachytherapy is often applied to oral cancers, with good outcomes and minimal loss of oral function. Especially, mold brachytherapy is best suited for superficial oral cancers with little or no bone invasion, such as gingival cancer, palatal mucosal cancer, or buccal mucosal cancer because of the thin tissue thickness that needs to be irradiated. A few cases have been reported on mold brachytherapy for gingival cancers, especially with high-dose-rates (HDR), these treatments were performed during hospitalization. We report a case of lower gingival squamous cell carcinoma (SCC) treated with HDR mold brachytherapy in an outpatient setting. A 71-year-old male with lower gingival SCC had received HDR mold brachytherapy ( $54 \mathrm{~Gy}, 9$ fractions, 5 days) as an outpatient. Eighteen months after the mold therapy, the patient had no recurrence and no metastasis. A search of the literature revealed no previous report of a case of gingival cancer treated with HDR mold brachytherapy in an outpatient.
\end{abstract}

Keywords: gingival cancer; mold brachytherapy; radiation therapy; outpatient

\section{Introduction}

Brachytherapy is often applied to oral cancers, with good outcomes and minimal loss of oral function [1]. Brachytherapy is classified into interstitial brachytherapy, intracavity brachytherapy, and mold brachytherapy. The main advantage of mold brachytherapy is that it is a non-invasive procedure. However, it is limited by the need for custom molds to be made for each individual and has a narrow range of applications. In the oral cavity, gingival cancer, palatal mucosal cancer, or buccal mucosal cancer are best suited for this method because of the thin tissue thickness that needs to be irradiated [2]. A shielded room is required during irradiation, and the treatment method has been used under hospitalization to date. A few cases have been reported on mold brachytherapy for gingival cancers $[3,4,5]$, especially with high-dose-rates (HDR), these treatments were performed during hospitalization [6,7]. 
In this report, we describe a case of lower gingival squamous cell carcinoma (SCC) that was successfully managed with HDR mold brachytherapy as outpatient.

\section{Case}

A 71-year-old male visited our hospital with the chief complaint of pain in the mucosa of the left lower gingiva. There was no comorbidity, but he had been diagnosed with severe anxiety disorder. Due to his anxiety disorder, he refused surgery and chemotherapy, and hospitalization. A mass was found in the mucosal area from the left lower gingiva to the retromolar trigone. The long and short diameters of the mass were $24 \mathrm{~mm}$ and $12 \mathrm{~mm}$, respectively, and the mass was mobile (Figure 1). The surface mucosa was irregular, and some ulceration was observed. The mass showed a thickness of $3 \mathrm{~mm}$ without bone destruction in the CT images (Figure 2). Clinically, there was no cervical lymphadenopathy and US, MRI and PET-CT showed no metastasis in the cervical lymph nodes and no distant metastasis. Based on the biopsy and imaging results, the patient was diagnosed with SCC (cT2NOM0, Stage II). Considering that the lesion was localized within the mucosa, we decided to perform HDR mold brachytherapy in an outpatient setting.

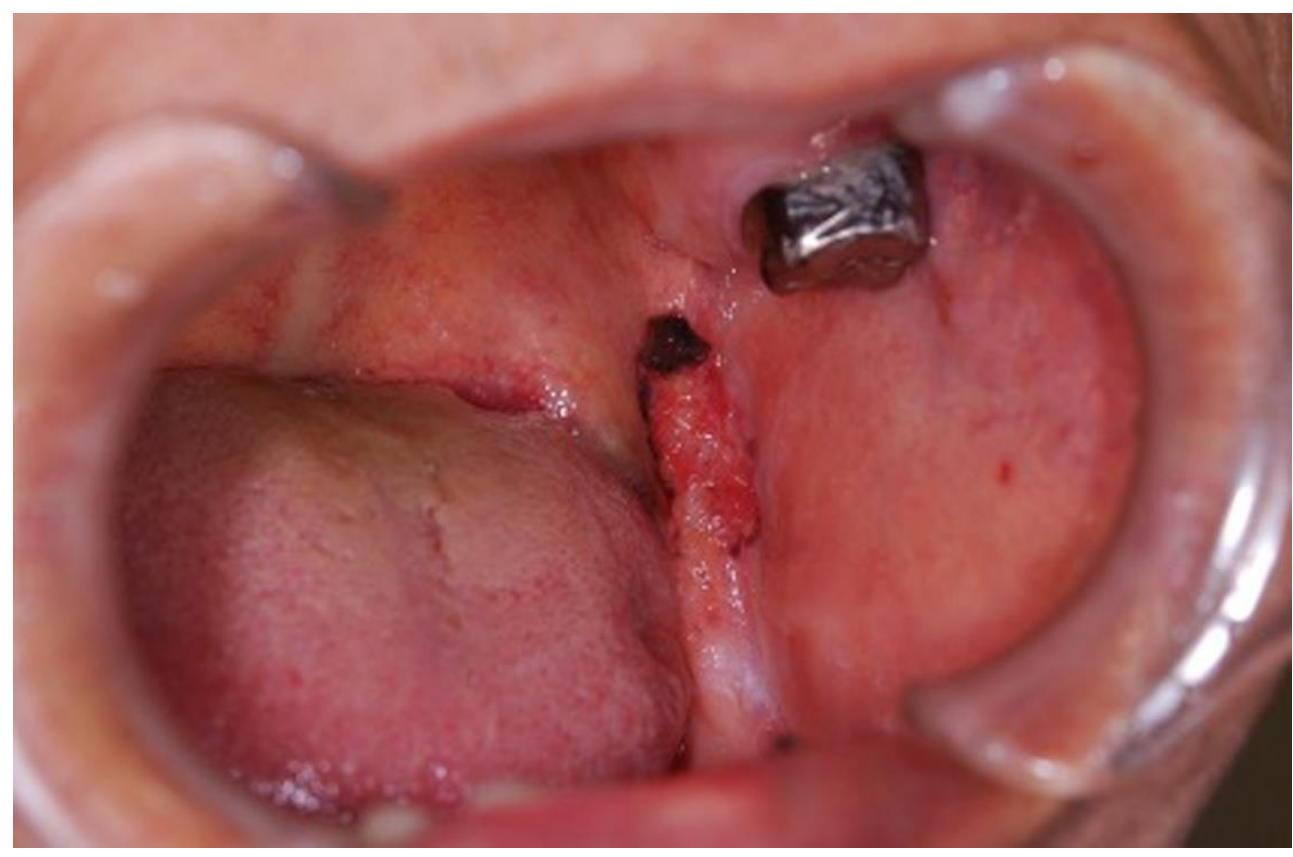

Figure 1. Pretreatment clinical photograph, showing a massive lesion from the left lower gingiva to the retromolar trigone, measuring $24 \times 12 \times 3 \mathrm{~mm}$; cT2N0M0, Stage II.
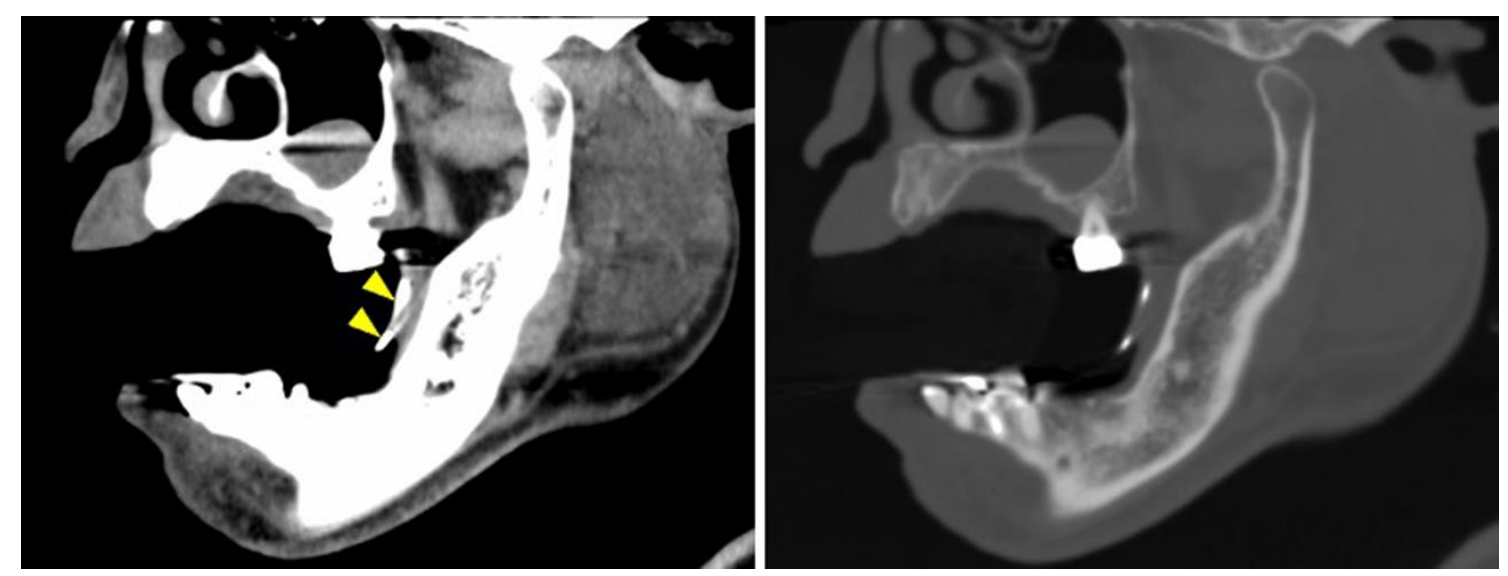

Figure 2. Pretreatment CT images. Representative sagittal section through the mandibular posterior body. (Left) Soft tissue kernel reconstruction. The arrowheads show one of three flexible applicator 
tubes used for HDR mold brachytherapy. The $3 \mathrm{~mm}$ region between the flexible applicator tube and the mandibular ramus corresponds to the tumor's thickness. (Right) The bone kernel reconstruction CT section shows an intact mandibular cortex in contact with the tumor, with no evidence of bone destruction.

An alginate impression of the mandible, including the lesion, was taken. A plaster model was made, and the location of the tumor was marked (Figure 3). A plastic sheet (0.5 $\mathrm{mm}$ thin) was placed in the tumor area, and three flexible applicator tubes to be used for HDR interstitial brachytherapy were placed and fixed with resin (Figure 4). To reduce the radiation exposure of the surrounding area, a 6-mm thick lead mold was made and placed on top of the plastic mold to create a two-layered mold (Figure 4).

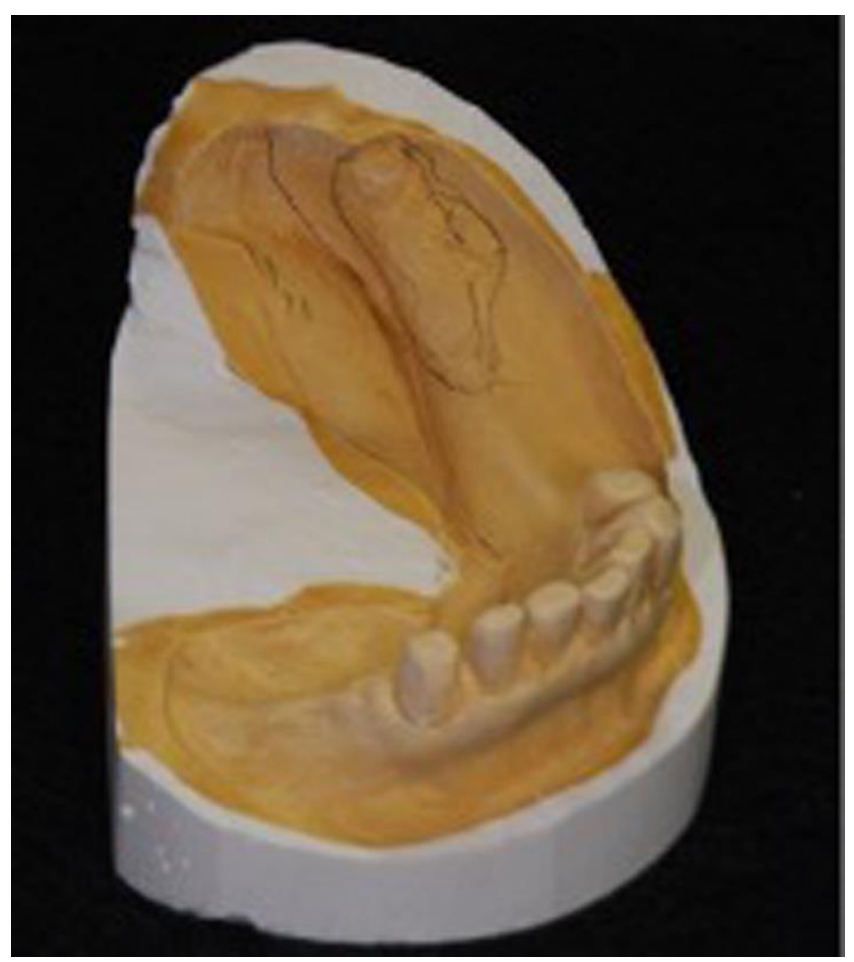

Figure 3. Plaster model. Note the region of the tumor outlined from the left lower gingiva to the retromolar trigone.
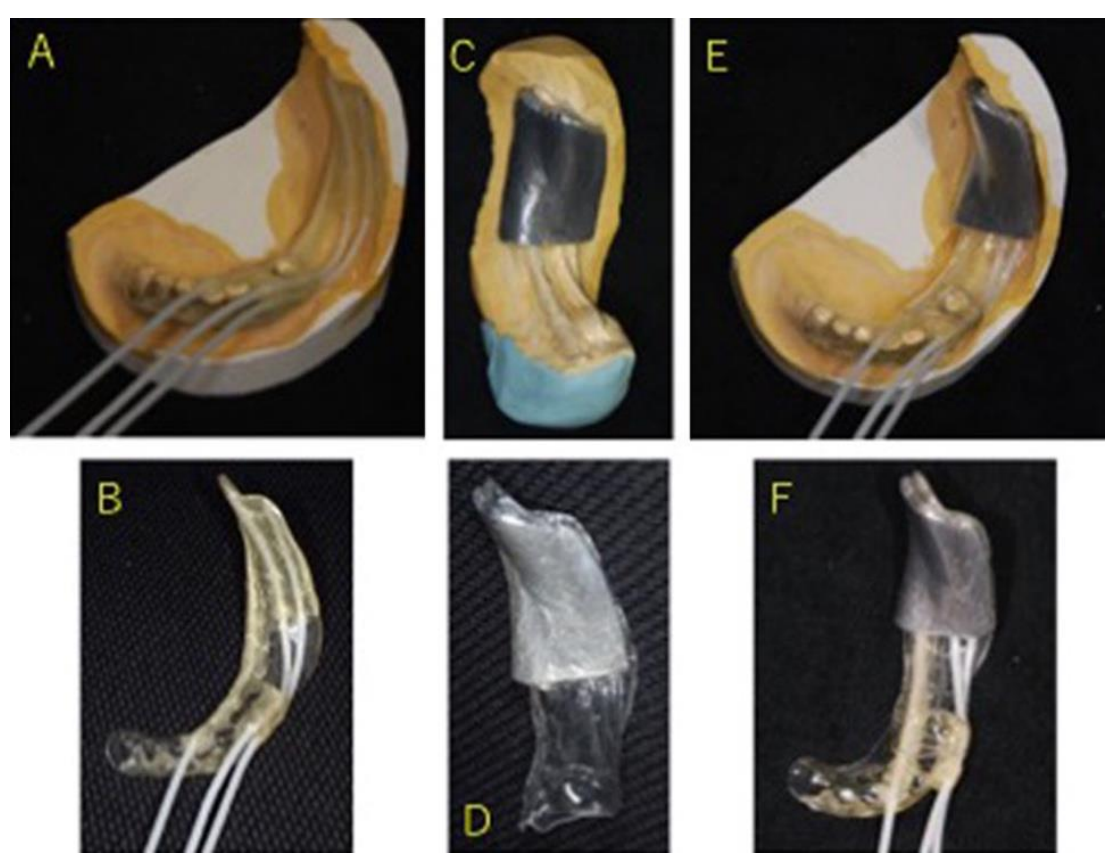
Figure 4. Steps of creating a mold. (A,B) The first plastic mold with three flexible applicator tubes attached. (C,D) Another mold made of lead to protect against exposure to radiation. (E,F) The twolayered mold.

In order to avoid metallic artifacts caused by lead, only the first mold was placed in the mouth; then, a CT scan was acquired and a three-dimensional treatment plan was performed using Oncentra Brachy (Elekta $\mathrm{AB}$, Stockholm, Sweden). The contour of the clinical target volume (CTV) was identified using the CT images. The planning target volume (PTV) was defined as an equivalent to CTV. We delivered $6 \mathrm{~Gy}$ as D100, i.e., minimum dose of the prescribed dose that was given to $100 \%$ of the PTV. The dose-distribution diagram is shown in Figure 5. Although no bone destruction was observed in the CT images, the PTV was designed to cover the gross tumor volume and to irradiate from the surface of the mandible to approximately $1 \mathrm{~cm}$ into the mandible. In addition, we also considered dose reduction to the normal tissues surrounding the tumor.
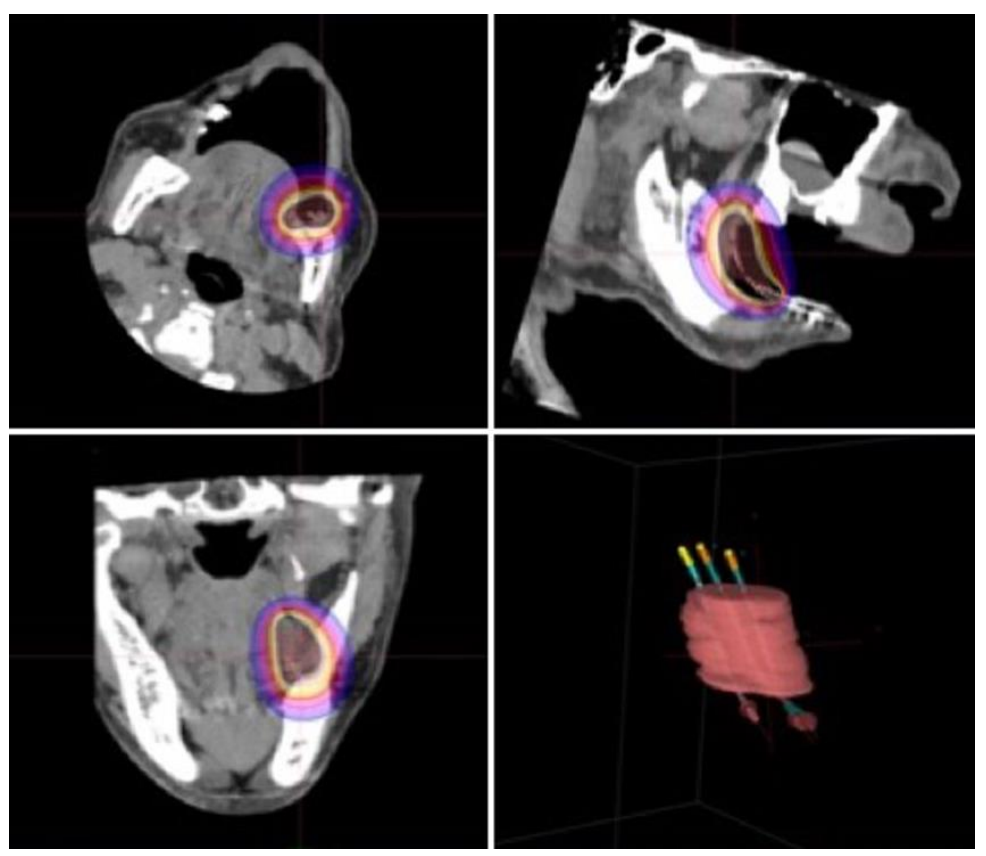

Figure 5. Dose-distribution diagram for HDR mold brachytherapy. Axial (top left), sagittal (top right) and coronal (bottom left) sections showing the dose distribution profile. A three-dimensional rendering image showing position of three flexible applicator tubes in relation to planning target volume (bottom right).

The two-layered mold was placed in the oral cavity, and irradiation started (Figure 6). The irradiation device was a microSelectron HDR-V2 (Elekta, Stockholm, Sweden), and the radiation source was 192-Ir. The total prescribed dose was set at 54 Gy per nine fractions at an interval of 6 hours or more in 5 days. 

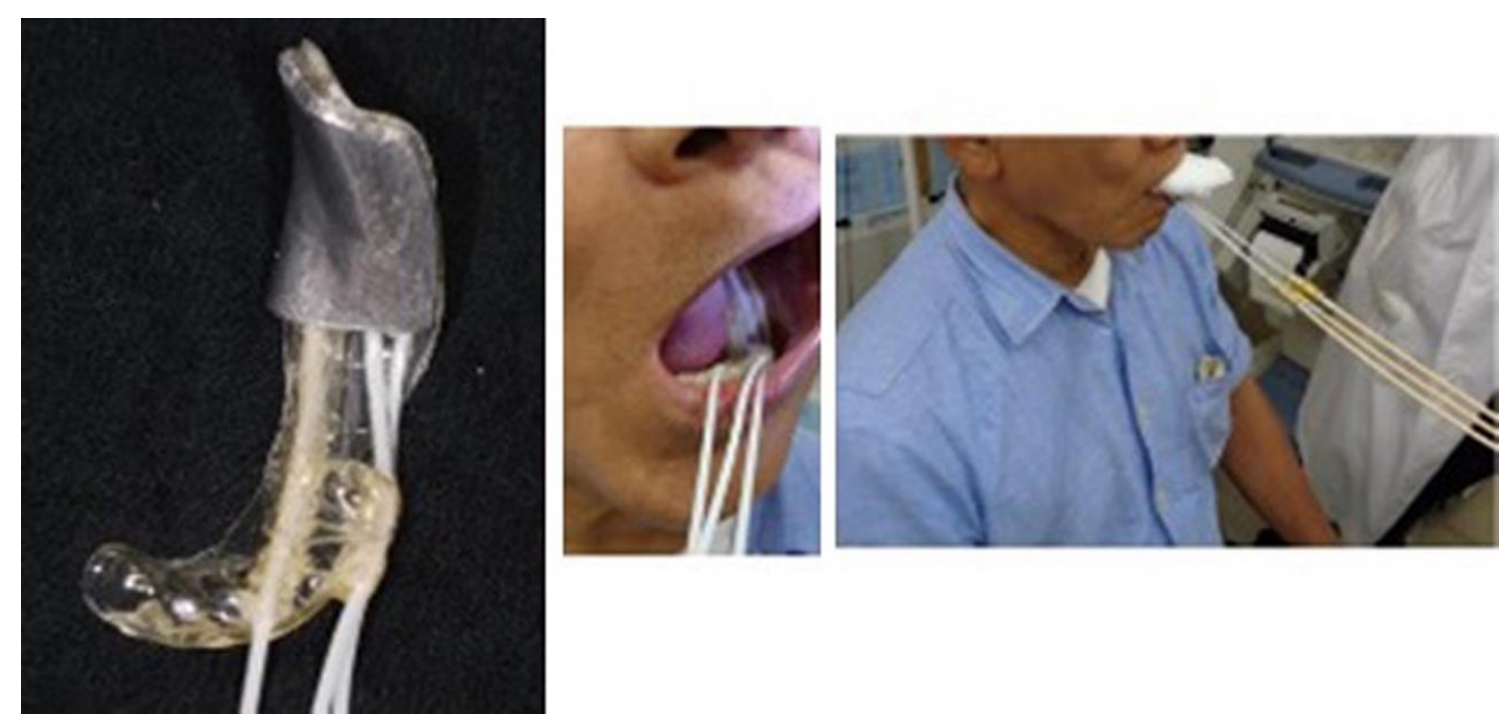

Figure 6. Treatment setup.

Although lead shielding was used, radiation-related stomatitis occurred immediately after the irradiation of the gingiva, buccal mucosa, and left lateral border of tongue other than the primary tumor. This stomatitis continued for 2 months after the irradiation was complete (Figures 7-9).

Dysgeusia and xerostomia were not reported. Imaging examinations were performed 6 months and 18 months post-treatment, and no abnormalities were found in the primary site of the lesion, nor were there any cervical lymph node metastases or distant metastases.

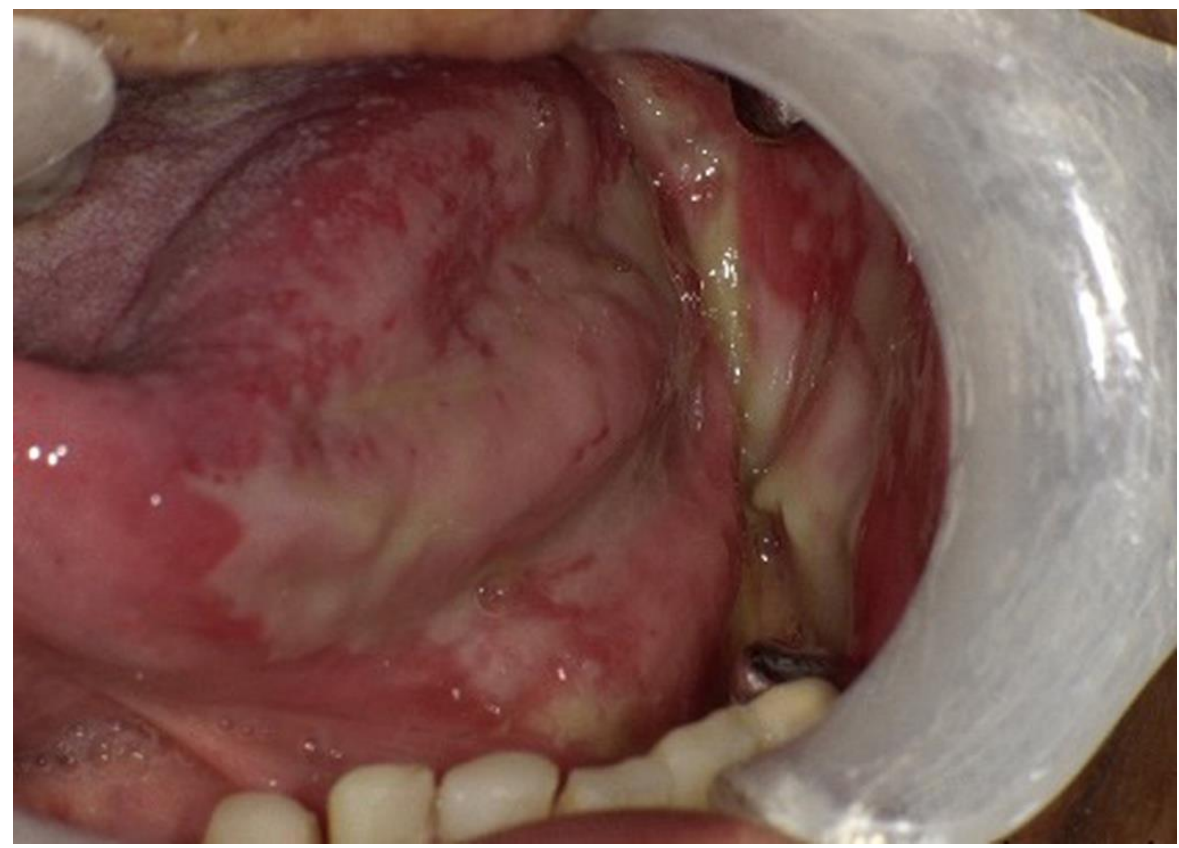

Figure 7. Clinical photograph after 1 week of treatment. Note mucositis on the left lateral border of tongue, floor of the mouth and cheek, adjacent to the region of the mold. 


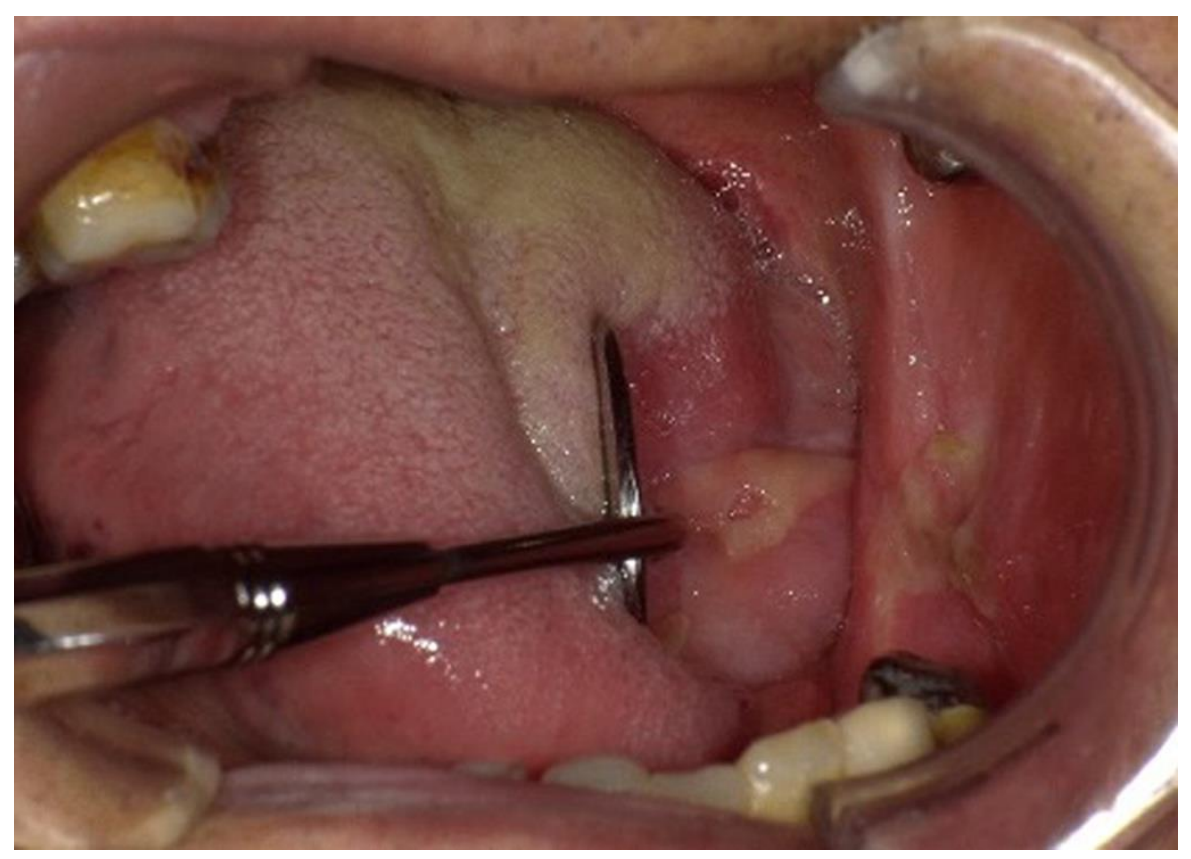

Figure 8. Clinical photograph after 2 months of treatment.

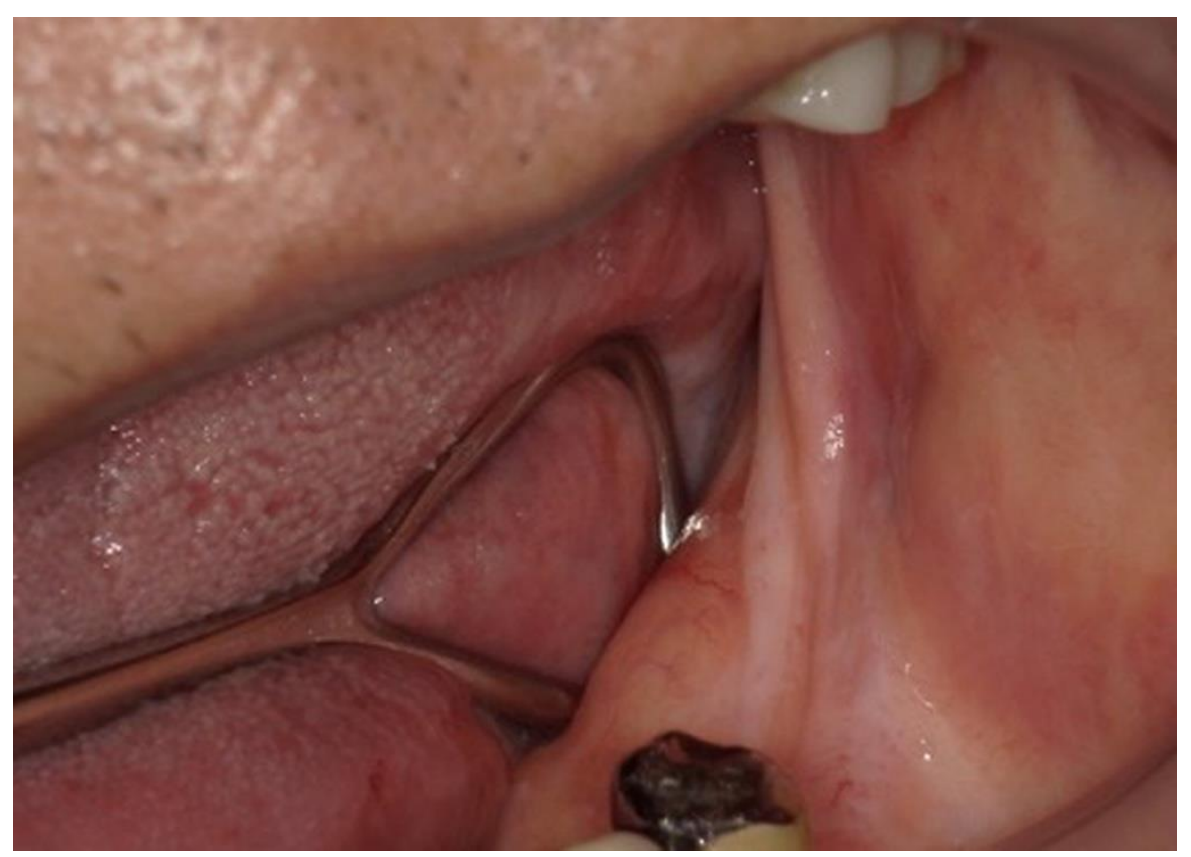

Figure 9. Clinical photograph after 12 months of treatment.

\section{Discussion}

Surgical resection is the standard therapeutic approach to manage patients with gingival cancer [8]. Alternatively, radiation therapy may be performed for superficial gingival cancer with little or no bone invasion. Radiation therapy can be classified into externalbeam radiation therapy and internal radiation therapy. Although the application of external-beam radiation therapy was initially considered for the patient, he refused it, fearing adverse effects such as wide-range mucositis and skin damage. Therefore, we chose mold brachytherapy from the internal radiation therapy options.

The greatest advantage of mold brachytherapy is that it eliminates the need for invasive procedures [1]. Since no applicator tube is inserted into the tissue, the tissue morbidity is reduced. In addition, the mold is removable, and the applicator tube only needs to be placed when the treatment is performed. In fact, there have been some reports of the mold method applied to treat superficial gingival cancer under hospitalization with local 
control and good prognosis [6,7]. However, to our knowledge, there have been no reports of mold therapy for gingival cancer, delivered in outpatient settings. We found no major problems with the reported outpatient treatment, and we believe that outpatient visits will be also feasible for mold brachytherapy in superficial gingival cancer cases in the future. Although it has been reported that mold brachytherapy is not suitable for the retromolar trigone because of the difficulty of covering [9], it may, to the contrary, be useful even in the retromolar trigone, if the tumor can be covered by the mold as this case.

In the present case, there were no teeth near the tumor area, and the mold was easy to fabricate and place in the region of interest (Figure 10). Since the tumor was mobile during palpation and the $\mathrm{CT}$ images showed no bone invasion, it was thought that there was no bone invasion. However, it is virtually impossible to irradiate only the tumor, and thus, the PTV was covered from the tumor surface to approximately $1 \mathrm{~cm}$ into the mandible. This dose prescription also took into account the risk of micro bone invasion of the tumor, meaning that all layers of bone cortex and only a part of the bone marrow were irradiated. On the other hand, the panoramic examination did not identify teeth in the irradiation field with a poor prognosis that would require extraction prior to mold brachytherapy. Thus, the possibility of future radiation-induced osteonecrosis of the jaw was considered to be low.

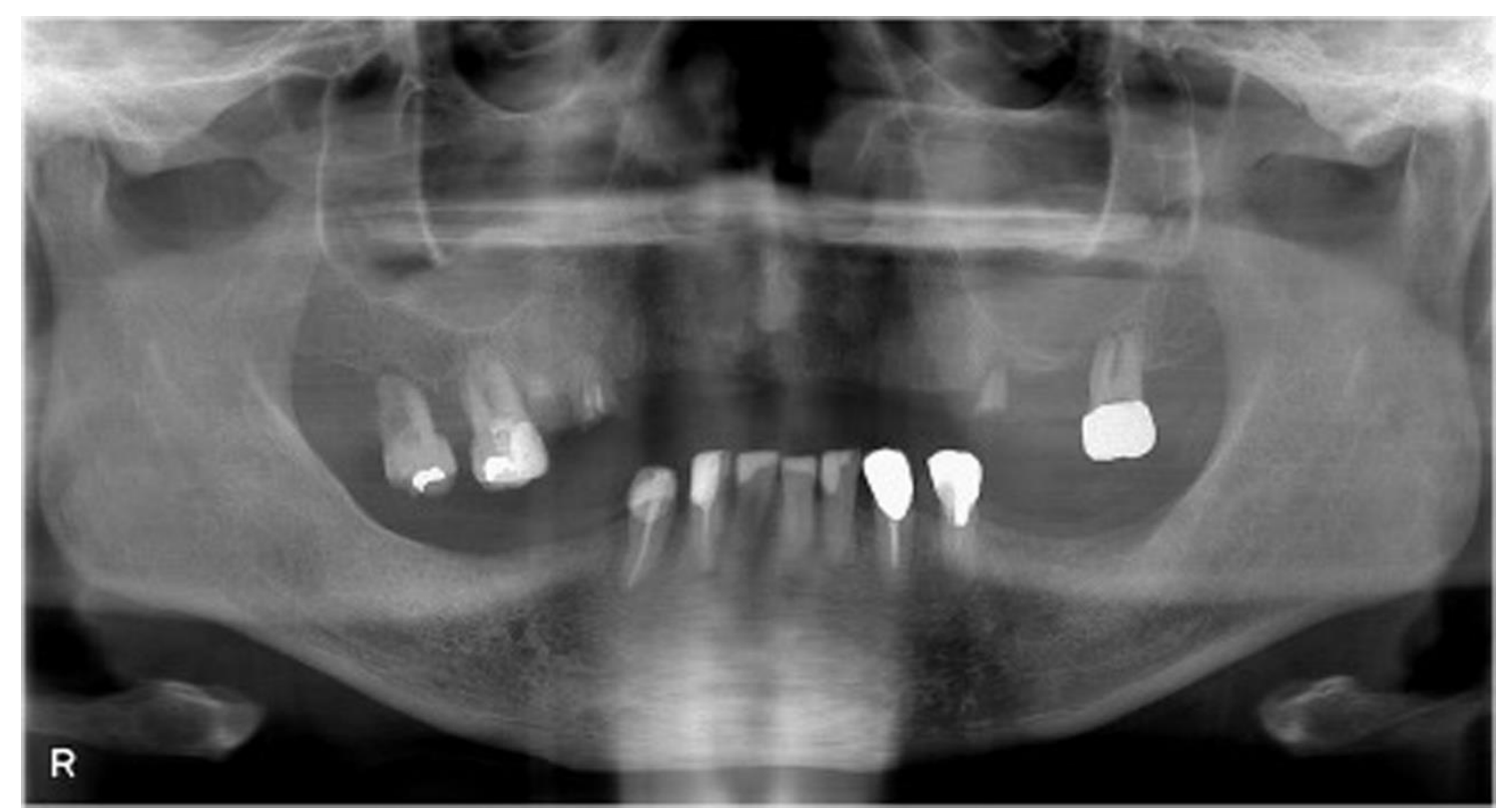

Figure 10. Panoramic pretreatment image.

Although the follow-up period was only about 18 months, no local recurrence, lymph-node metastasis, or distant metastasis was observed. It is promising that no bone changes in the irradiation field have been observed to date. As the patient population ages, it is expected that ever more patients will become inoperable due to comorbidity or will refuse hospitalization. Although the target population is limited, the described outpatient mold brachytherapy provides an attractive option that is less invasive and less burdensome for certain oral cancer patients in the future.

\section{Conclusions}

We provide the first report of a case of lower gingival SCC treated with HDR mold brachytherapy in an outpatient setting.

Author Contributions: Study design, A.T., Hiroaki Shimamoto, Y.U., and S.M.; conceptualization, S.M.; software, M.O.; data collection, A.T., Hiroaki Shimamoto, Y.U., T.T., T.M., and M.O.; data analysis, S.K., S.M.M., T.H., K.O., and Hiroya Shiomi; writing-original draft preparation, A.T., Hiroaki Shimamoto, Y.U., T.T., and T.M.; writing - review and editing, S.K., S.M.M., T.H., K.O., and Hiroya 
Shiomi; supervision, Hiroaki Shimamoto.; project administration, S.M. All the authors have read and agreed to the published version of the manuscript.

Funding: This research received no external funding.

Institutional Review Board Statement: The local Ethics Committee of the Osaka University Graduate School of Dentistry does not require ethical approval for reporting this type of case, in view of the retrospective nature of the study and the fact that all of the procedures were performed as part of routine care. This study followed the principles of the Declaration of Helsinki.

Informed Consent Statement: Written informed consent was obtained from the patient for his anonymized information to be published in this article.

Data Availability Statement: The data presented in this study are available on request from the corresponding author.

Conflicts of Interest: The authors declare no conflict of interest.

\section{References}

1. Unetsubo, T.; Matsuzaki, H.; Takemoto, M.; Katsui, K.; Hara, M.; Katayama, N.; Waki, T.; Kanazawa, S.; Asaumi, J. High-doserate brachytherapy using molds for lip and oral cavity tumors. Radiat. Oncol. 2015, 10, 81.

2. Prisciandaro, J.I.; Foote, R.L.; Herman, M.G.; Lee, S.J.; LaJoie, W.N.; van Blarcom, A.B.; Yeakel, P.D. A buccal mucosa carcinoma treated with high dose rate brachytherapy. J. Appl. Clin. Madical Phys. 2005, 6, 8-12.

3. Wong, G.; Cirino, E.T.; Ladd, R.O.; Halvorsen, P.H.; Iftimia, I. Use of customized intraoral mold high-dose-rate brachytherapy in the treatment of oral cavity cancer in an elderly patient. Pract. Radiat. Oncol. 2015, 5, 74-78.

4. Wong, G.; Cirino, E.T.; Ladd, R.O.; Halvorsen, P.H.; Iftimia, I. Treatment of upper gum carcinoma with high-dose-rate customized-mold brachytherapy. Brachytherapy 2008, 7, 267-269.

5. Cengiz, M.; Ozyar, E.; Ersu, B.; Akyol, F.H.; Atahan, I.L. High dose rate mold brachytherapy of early gingival carcinoma: A clinical report. J. Prosthet. Dent. 1999, 82, 512-514.

6. Obinata, K.; Ohmori, K.; Shirato, H.; Nakamura, M. Experience of high-dose-rate brachytherapy for head and neck cancer treated by a customized intraoral mold technique. Radiat. Med. 2007, 25, 181-186.

7. Ariji, E.; Hayashi, N.; Kimura, Y.; Uchida, T.; Hayashi, K.; Nakamura, T. Customized mold brachytherapy for oral carcinomas through use of high-dose-rate remote afterloading apparatus. Oral. Surg. Oral. Med. Oral. Pathol. Oral. Radiol. Endod. 1999, 87, 508-512.

8. NCCN Clinical Practice Guidelines in Oncology Head and Neck cancers version 1.2022. 2021. Available online: https://www.nccn.org (accessed on 21th February 2022).

9. Nagata, Y.; Okajima, K.; Hiraoka, M.; Yokoe, Y.; Nishida, M.; Nishimura, Y. High-dose-rate brachytherapy using molds for oral cavity cancer: The technique and its limitations. Int. J. Clin. Oncol. 1998, 3, 351-356. 